Article

\title{
$(T ; N)$ and Residual Fuzzy Co-Implication in Dual Heyting Algebra with Applications
}

\author{
Iqbal H. Jebril \\ Department of Mathematics, Science Faculty, Taibah University, Medina, Saudi Arabia; \\ iqbal501@hotmail.com; Tel.: +966-590-638-501
}

\begin{abstract}
Recently, many authors have been interested to introduce fuzzy implications over t-norms and t-conorms. In this paper, we introduce $(S, N)$ and residuum fuzzy implication for Dubois t-norm and Hamacher's t-norm. Also, new concepts so-called $(T, N)$ and residual fuzzy co-implication in dual Heyting Algebra are investigated. Some examples as well as application are discussed as well.
\end{abstract}

Keywords: Fuzzy implications; $(\mathrm{S}, \mathrm{N})$ implication; residuum t-norm; $(T, \mathrm{~N})$ co-implication; residual co-implication

PACS: J0101

\section{Introduction}

In fuzzy logic, the basic theory of connective AND $(\wedge)$, OR $(\vee)$, NOT $(\neg)$ are often modeled as (strong negations, t-norm, and t-conorms). An important notion in fuzzy set theory is that of t-norm $(T)$, t-conorms $(S)$ and strong negations $\left(N_{C}\right)$ that are used to define a generalized intersection, union and negation of fuzzy sets (see [5] and [6]). Implication and co-implication functions play an important notion in fuzzy logic, approximate reasoning, fuzzy control, intuitionistic fuzzy logic and approximate reasoning of expert system (see ([1], [2], [3], [4], [7], [8], and [9]).

The notion of t-norm and t-conorm turned out to be basic tools for probabilistic metric spaces (see [10] an [11]), but also in several other parts and have found diverse applications in the theory of fuzzy sets, fuzzy decision making, in models of certain many-valued logics or in multivariate statistical analysis. (see [12], [13], and [10]).

\section{$2 \quad$ Preliminaries}

The logic connectives like negation $\neg$ is interpreted by a strong negation, conjunction $\wedge$ by a triangular norm and disjunction $\vee$ by triangular conorm. [14] 


\subsection{Triangular Norm and Triangular conorm}

The conjunction $\wedge$ in fuzzy logic, it is often modeled as follows:

Definition 2.1. [10]: A mapping $T$ from $[0,1]^{2}$ into $[0,1]$ is a triangular norm (in short, t-norm), iff $T$ are commutative, nondecreasing in both arguments, associative and which satisfies $T(p, 1)=p, \forall p \in[0,1]$.

Also, disjunction $\vee$ in fuzzy logic is often modeled as follows:

Definition 2.2. [10] A mapping $S$ from $[0,1]^{2}$ into $[0,1]$ is a triangular conorm (in short, t-conorm), iff $S$ are commutative, nondecreasing in both arguments, associative and which satisfies $S(p, 0)=p, \forall p \in[0,1]$.

Proposition 2.1. [10] A mapping $S$ is a triangular conorm iff there exists a triangular norm $T$ such that $S(p, q)=1-T(1-p, 1-q), \forall p, q \in[0,1]$. In this case $S$ is called the dual t-conorm of $T$.

The standard examples of t-norm and dual t-conorms are stated in the following:

\begin{tabular}{|c|c|}
\hline t--norm $(T)$ & Dual t-conorm $(S)$ \\
\hline $\begin{array}{c}M(p, q)=\min (p, q) \\
\text { (Minimum t-norm) }\end{array}$ & $\begin{array}{l}\mathrm{S}_{M}(p, q)=\max (p, q) \\
\text { (Maximum t-conorm) }\end{array}$ \\
\hline $\begin{array}{c}\prod(p, q)=p q \\
\text { (Probabilistic Product t-norm) }\end{array}$ & $\begin{array}{c}\mathrm{S}_{\Pi}(p, q)=p+q-p q, \\
\text { (Probabilistic sum t-conorm) }\end{array}$ \\
\hline $\begin{array}{l}W(p, q)=\left\{\begin{array}{l}p \text { if } q=1 \\
q \text { if } p=1 \\
0 \text { if } p, q \in[0,1)\end{array}\right. \\
\text { (Drastic or weak t-norm) }\end{array}$ & $\begin{array}{l}S_{W}(p, q)= \begin{cases}p & \text { if } q=1, \\
q & \text { if } p=1 \\
1 & \text { otherwise. }\end{cases} \\
\text { (Drastic or largest t-conorm) }\end{array}$ \\
\hline $\begin{array}{c}N(p, q)= \begin{cases}\min (p, q) & \text { if } p+q \geq 1 \\
0 & \text { if } p+q<1 .\end{cases} \\
\text { (Nilpotent t-norm) }\end{array}$ & $\begin{array}{c}\mathrm{S}_{N}(p, q)=\left\{\begin{array}{cc}\max (p, q) & \text { if } p+q<1, \\
0 & \text { if } p+q \geq 1 .\end{array}\right. \\
\text { (Nilpotent } \quad \text { t-conorm) }\end{array}$ \\
\hline $\begin{array}{l}L(p, q)=\max (p+q-1,0) \\
(\text { Lukasiewicz t-norm) }\end{array}$ & $\begin{array}{l}\mathrm{S}_{L}(p, q)=\min (p+q, 1), \\
\text { (Bounded Sum t-conorm) }\end{array}$ \\
\hline
\end{tabular}




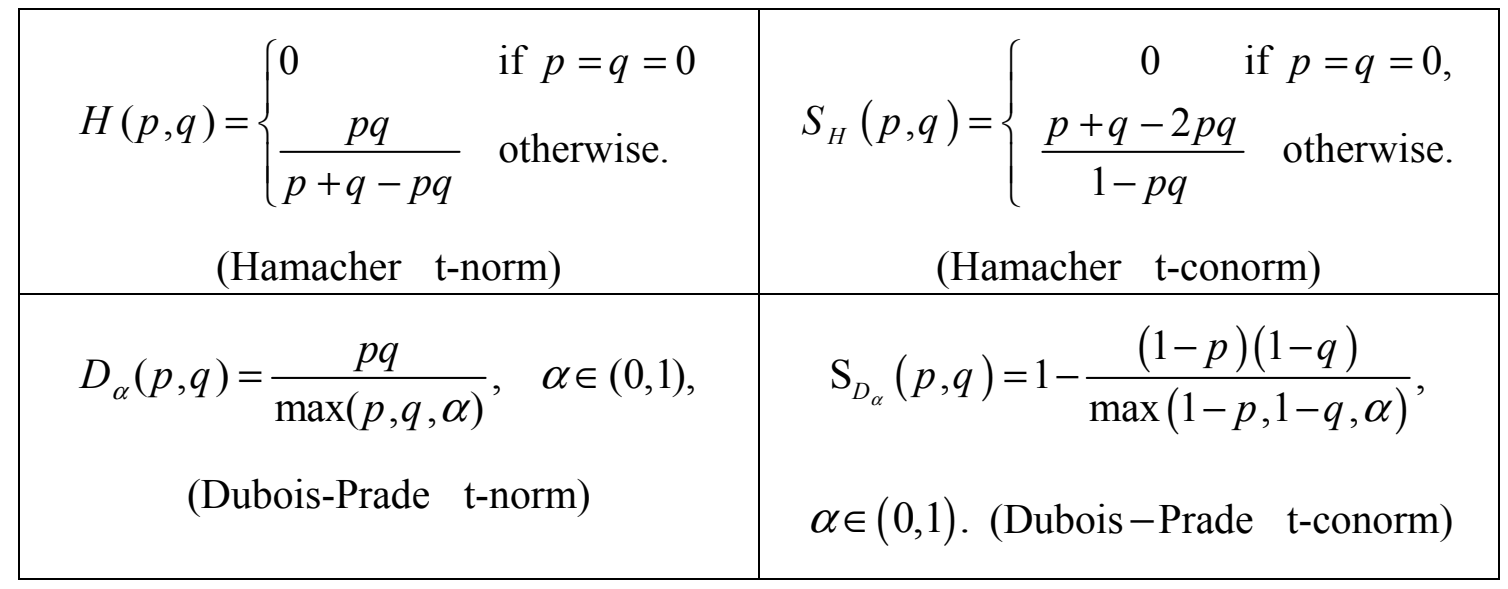

For other family of t-norm (not needed here) we refer the reader to [12] for instance.

If $T_{1} \leq T_{2}$ and there is at least one pair $(p, q) \in[0,1]^{2}$ such that $T_{1}(p, q)<T_{2}(p, q)$ then we briefly write $T_{1}<T_{2}$. With this, the above t-norms satisfy the next known chain of inequalities

$$
W<L<\Pi<H<M .
$$

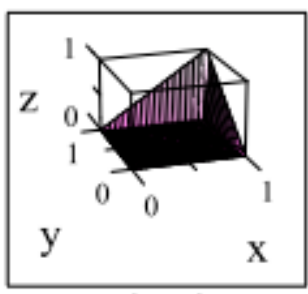

$W(p, q)$

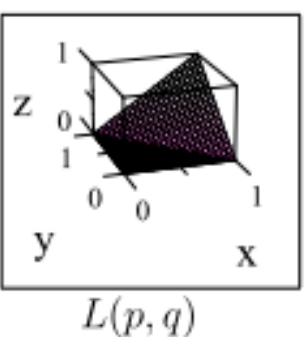

$L(p, q)$

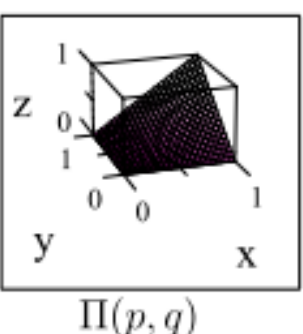

$\Pi(p, q)$

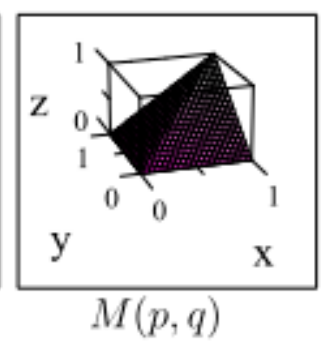

$M(p, q)$

Two t-norm $T_{1}$ and $T_{2}$ are called comparable if $T_{1} \leq T_{2}$ or $T_{2} \leq T_{1}$ holds. The above chain of inequalities shows that $W, L, \Pi, H$ and $M$ are comparable. It is not hard to see that , for example, $\Pi$ and $N$ are not comparable, while $W, N$ and $M$ comparable with

$$
W<N<M \text {. }
$$

\subsection{Negation Function}

The truth table of the classical negation $N$ is given in following table.

\begin{tabular}{|c|c|}
\hline$p$ & $N p$ \\
\hline 0 & 1 \\
\hline 1 & 0 \\
\hline
\end{tabular}

Definition 2.3. [15] A mapping $N$ from $[0,1]$ into $[0,1]$ is a negation function, iff: 
1. $N(0)=1, N(1)=0$;

2. $N(p) \leq N(q)$, if $p \geq q . \forall p, q \in[0,1]$. (Monototonicity)

A negation function is strict, iff:

1. $N(p)$ is continuous;

2. $N(p)<N(q)$, if $p>q . \forall p, q \in[0,1]$.

A strict negation function is strong or volutive, iff:

1. $N(N(p))=p, \forall p \in[0,1]$.

A negation function is weak, iff $N$ is not strong.

Example 2.1. [15] The strong negation $\left(N_{C}(p)=1-p\right)$, strict negation but not strong $\left(N_{k}(p)=1-p^{2}\right)$, weaker negation $\left(N_{D_{1}}(p)=\left\{\begin{array}{l}1 \text { if } p=0, \\ 0 \text { if } p>0 .\end{array}\right)\right.$ and strongest negation $\left(N_{D_{2}}(p)=\left\{\begin{array}{l}1 \text { if } p<1 \\ 0 \text { if } p=1\end{array}\right)\right.$.

Definition 2.4. [13] Let $T$ be a t-norm and $S$ be a t-conorm. A mapping $N_{T}, N_{S}$ from $[0,1]$ into $[0,1]$ defined by

$$
\begin{aligned}
& N_{T}(p)=\sup \{r \in[0,1] \backslash T(p, r)=0\}, \text { for every } p \in[0,1], \\
& N_{S}(p)=\inf \{r \in[0,1] \backslash S(p, r)=1\}, \text { for every } p \in[0,1],
\end{aligned}
$$

are called the natural negation of $T$ and $S$, respectively.

\section{$3(T, \mathrm{~N})$ Co-Implication}

This section will be devoted to introduce the concept of $(T, N)$ co-implication. The relation between classical logic and fuzzy logic as well as some examples are also discussed

Definition 3.1. [15] A mapping $I$ from $[0,1]^{2}$ into $[0,1]$ is fuzzy implication if, $\forall p, q, r \in[0,1]$, the following conditions are satisfied: 
$I 1: I(1,1)=I(0,1)=I(0,0)=1$ and $I(1,0)=0$.

$I 2: I(p, q) \geq I(r, q)$ if $p \leq r$.

$I 3: I(p, q) \leq I(p, r)$ if $q \leq \mathrm{r}$.

The set of all fuzzy implications is denoted by $F I$.

In classical logic, the main two ways to defining an implication 怰 in Boolean lattice $(L, \wedge, \vee, \neg)$ are $p \Rightarrow q \equiv \neg p \vee q$ and $p \Rightarrow q \equiv \max \{r / p \wedge r \leq q\}$. [18]

The $(S, N)$ implication and residual implication is generalization of these material implications to fuzzy logic.

Definition 3.2. [18] A mapping $I$ from $[0,1]^{2}$ into $[0,1]$ is called an $(S, N)$ implication if there exist a fuzzy negation $N$ and a t-conorm $S$ such that

$$
I_{S, N}(p, q)=S(N(p), q), \forall p, q \in[0,1] .
$$

Definition 3.3. [18] Let $T$ a left-continuous t-norm. Then, the residual implication or $R$-implication derived form $T$ is given by

$$
I_{T}(p, q)=\sup \{r \in[0,1] / T(r, p) \leq q\}, \quad \forall p, q \in[0,1] .
$$

i.e. $T(r, p) \leq q \Leftrightarrow r \leq I_{T}(p, q), \forall p, q, r \in[0,1]$.

Remark 3.1. [18] It easy to check that for every left-continuous t-norm $T$, the only operation $I_{T}(p, q)$ satisfies $(\mathrm{R})$ is called

$I_{T}(p, q)=\max \{r \in[0,1] / T(r, p) \leq q\}$,

where the right side exists $\forall p, q \in[0,1]$.

Definition 3.4. [19] A mapping $J$ from $[0,1]^{2}$ into $[0,1]$ is a fuzzy co-implication if, $\forall p, q, r \in[0,1]$, the following conditions are satisfied:

$J 1: J(1,1)=J(1,0)=J(0,0)=0$ and $J(0,1)=1$. 
$J 2: J(p, q) \geq J(r, q)$ if $p \leq r$.

$J 3: J(p, q) \leq J(p, r)$ if $q \leq r$.

The set of all fuzzy co-implication is denoted by $C o-F I$.

From the definition 3.4. $J(1, q)=J(p, 0)=0$ and $J(p, p)=0, \forall p, q \in[0,1]$.

Lemma 3.1. If a mapping $J$ from $[0,1]^{2}$ into $[0,1]$ satisfies $(J 1)$ and $(J 2)$, then the mapping $N_{J}:[0,1] \rightarrow[0,1]$ defined by

$$
N_{J}(p)=J(p, 1), \quad p \in[0,1],
$$

is a fuzzy negation.

The following properties are generalization of fuzzy implication and fuzzy co-implication from classical logic.

Definition 3.5. [18] A fuzzy implications $I$ and fuzzy co-implications $J$ is said to satisfy the following most important properties, $\forall p, q, r \in[0,1]$.

\begin{tabular}{|ll|ll|}
\hline$I(1, q)=q ;$ & $(\mathrm{NP})$ & $J(0, q)=q ;$ & $(\mathrm{Co}-\mathrm{NP})$ \\
\hline$I(p, I(q, r))=I(q, I(p, r)) ;$ & $(\mathrm{EP})$ & $J(p, J(q, r))=J(q, J(p, r)) ;$ & $(\mathrm{Co}-\mathrm{EP})$ \\
\hline$I(p, p)=1 ;$ & $(\mathrm{IP})$ & $J(p, p)=0 ;$ & $(\mathrm{Co}-\mathrm{IP})$ \\
\hline$I(p, q)=1 \Leftrightarrow p \leq q ;$ & $(\mathrm{OP})$ & $J(p, q)=0 \Leftrightarrow p \geq q$.
\end{tabular}

Co-implication are extensions of the Boolean co-implication $\not \measuredangle(p \not \measuredangle q$ meaning that $p$ is not necessary for $q$ ). (see [20])

Proposition 3.1. The operator 怾 (material co-implication) is generated by Boolean negation 憭 and conjunction 憭:

$$
q \not \nexists p \equiv q \wedge \neg p .
$$

The $(T, N)$ co-implication is generalization of this material co-implication to fuzzy logic. In the following table we can see the truth table for the classical co-implication

\begin{tabular}{|c|c|c|c|c|}
\hline$p$ & $q$ & $p \Rightarrow q$ & $q \not \nexists p$ & $q \wedge \neg p$ \\
\hline 0 & 0 & 1 & 0 & 0 \\
\hline 0 & 1 & 1 & 1 & 1 \\
\hline 1 & 0 & 0 & 0 & 0 \\
\hline
\end{tabular}




\begin{tabular}{|l|l|l|l|l|}
\hline 1 & 1 & 1 & 0 & 0 \\
\hline
\end{tabular}

Definition 3.6. A mapping $J$ from $[0,1]^{2}$ into $[0,1]$ is called an $(T, N)$ co_implication if there exists a t-norm $T$ and a fuzzy negation $N$ such that

$$
J_{T, N}(p, q)=T(q, N(p)), \quad \forall p, q \in[0,1] .
$$

A relation between fuzzy negations and $(S, N)$ implication is given in the next proposition.

Proposition 3.2 Let $J_{T, N}$ be an $(T, N)$ implication, then $N_{J_{T, N}}=N$.

Proof. For any $p \in[0,1], J_{T, N}(p)=J_{T, N}(p, 1)=T(1, N(p))=N(p)$.

Example 3.1. In the following examples we assume that $N_{C}$ is a strong negation.

t-norm

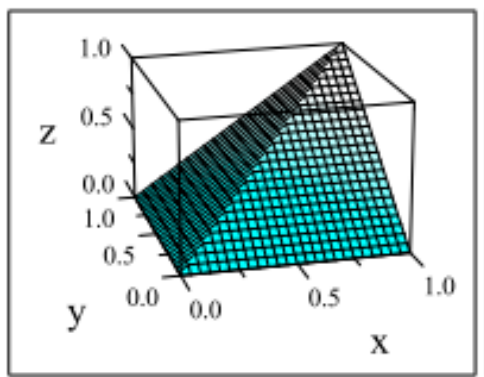

$M(p, q)$

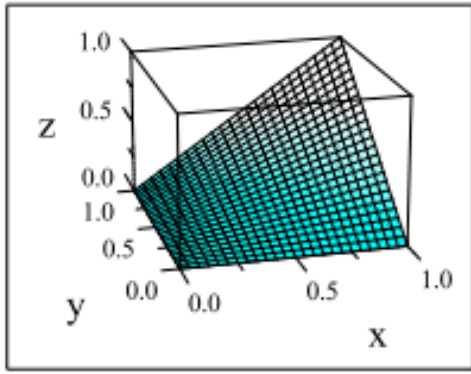

$\Pi(p, q)$

$$
J_{T, N_{C}}
$$

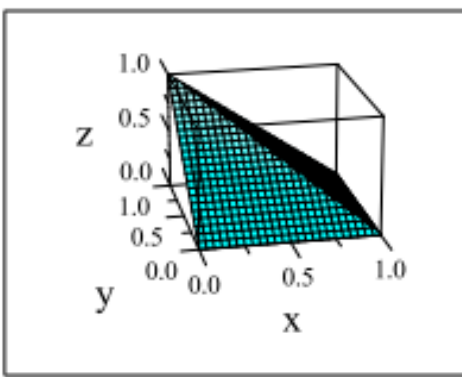

$J_{M, N_{C}}(p, q)=\min (q, 1-p)$

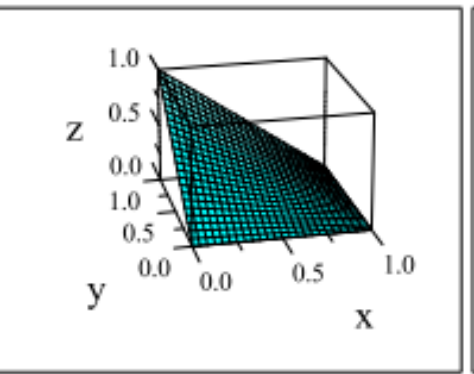

$$
J_{\Pi, N_{C}}(p, q)=q-p q
$$

$J_{T, N_{C}}$ and $I_{S, N_{C}}$

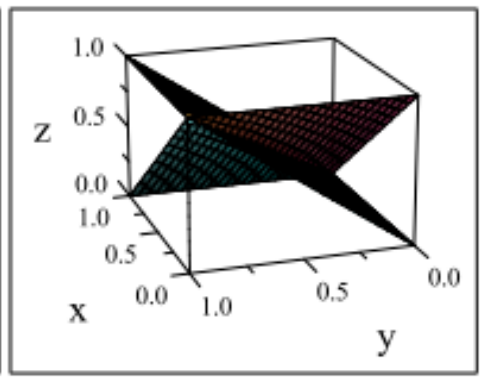

$$
J_{M, N_{C}} \text { and } I_{S_{M}, N_{C}}
$$

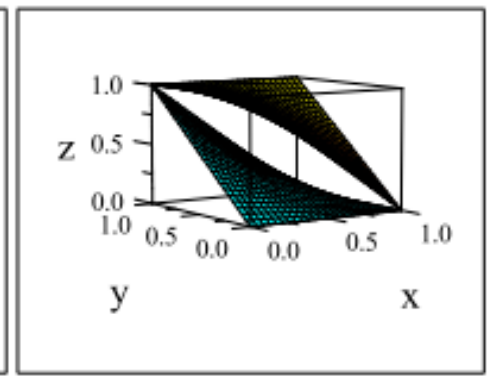

$J_{\Pi, N_{C}}$ and $I_{S_{\Pi}, N_{C}}$ 

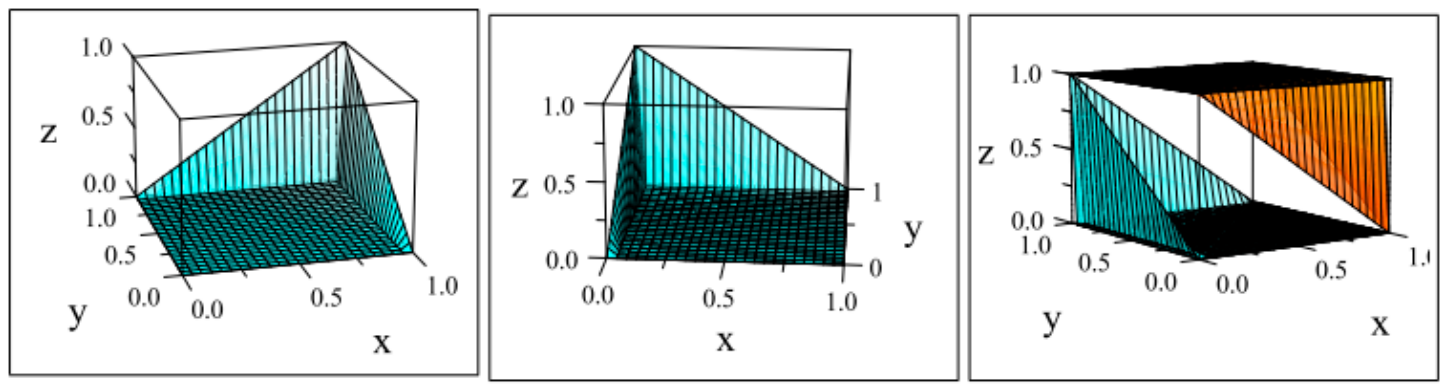

$$
W(p, q) \quad J_{W, N_{C}}(p, q)=\left\{\begin{array}{ll}
q & \text { if } q=1, \\
1-p & \text { if } q=1, \\
0 & \text { otherwise. }
\end{array} \quad J_{W, N_{C}} \text { and } I_{S_{W}, N_{C}}\right.
$$
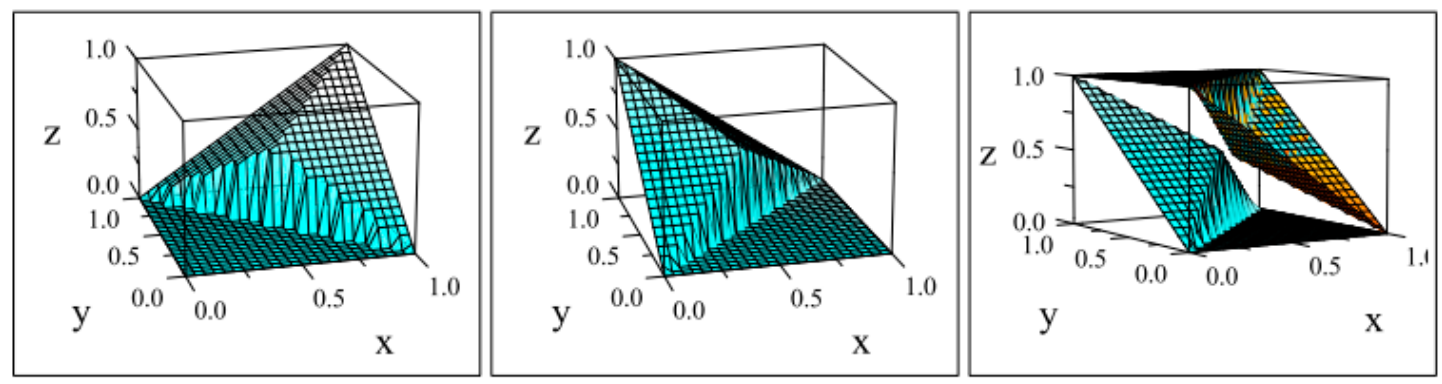

$$
N(p, q) \quad J_{N, N_{C}}(p, q)=\left\{\begin{array}{cl}
\min (q, 1-p) & \text { if } p<q, \\
0 & \text { if } p \geq q .
\end{array} \quad J_{N, N_{C}} \text { and } I_{S_{N}, N_{C}}\right.
$$
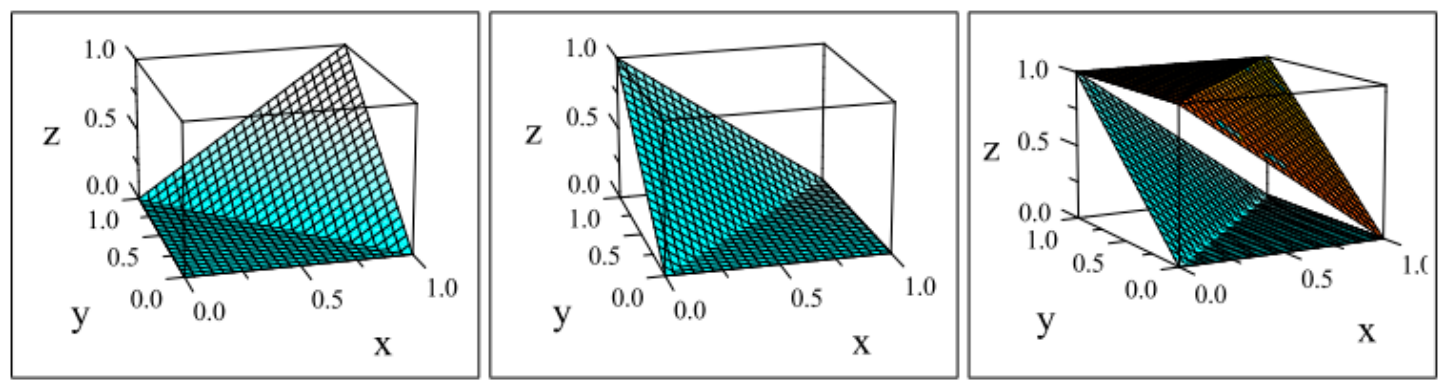

$$
L(p, q)
$$

$J_{L, N_{C}}(p, q)=\max (q-p, 0)$

$$
J_{L, N_{C}} \text { and } I_{S_{L}, N_{C}}
$$
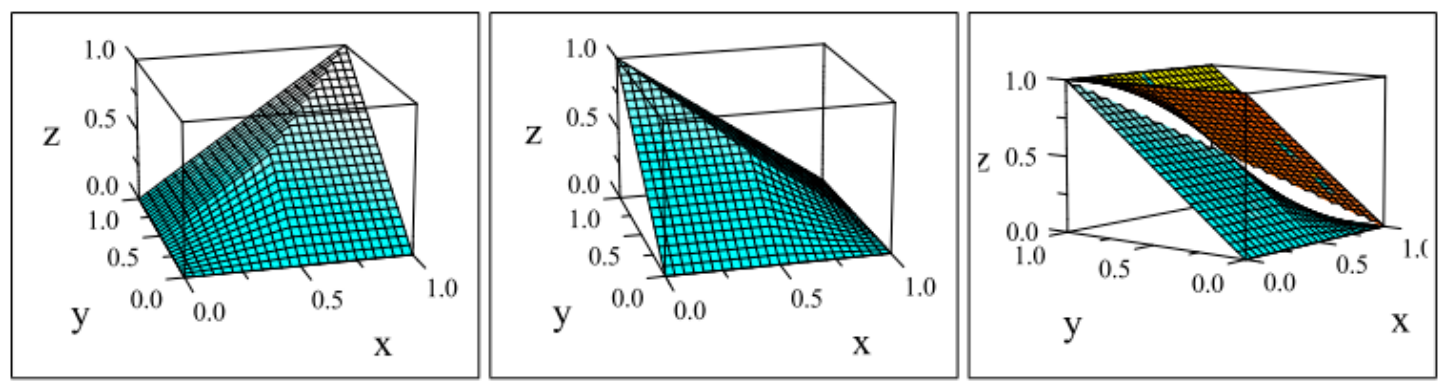

$$
H(p, q)
$$

$$
J_{H, N_{C}}(p, q)=\frac{q(1-p)}{1-p+q p}
$$

$J_{H, N_{C}}$ and $I_{S_{H}, N_{C}}$ 

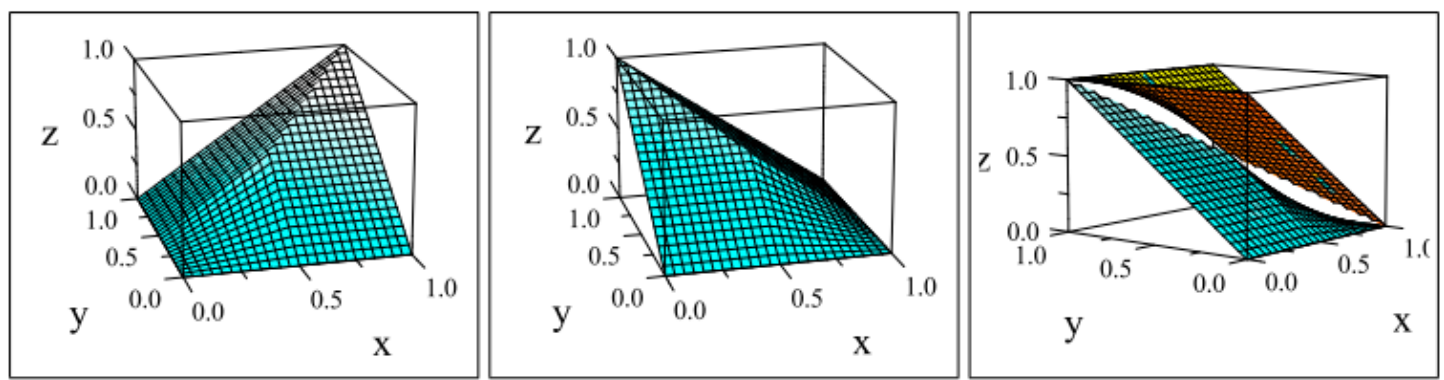

$D_{\alpha}(p, q), \alpha \in(0,1) \quad J_{D_{\alpha}, N_{C}}(p, q)=\frac{q(1-p)}{\max (q, 1-p, 0.5)} \quad J_{D_{\alpha}, N_{C}}$ and $I_{S_{D_{\alpha}}, N_{C}}$

Example 3.2. For t_norm $T$ and t-conorm $S$

1) A fuzzy negation $N_{2}(p)=1-p^{2}$, then, the basic $\left(S, N_{2}\right)$ implications and $I_{S_{M}, N_{2}}\left(T, N_{2}\right)$ co_implications $J_{M, N}$ are:

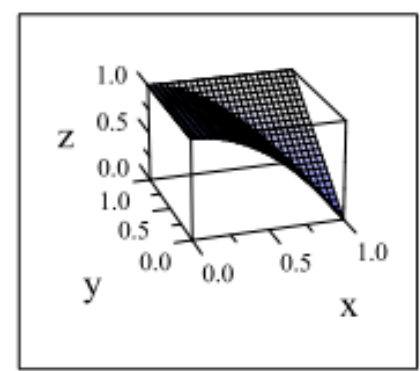

$I_{S_{M}, N_{2}}(p, q)=\max \left(1-p^{2}, q\right)$

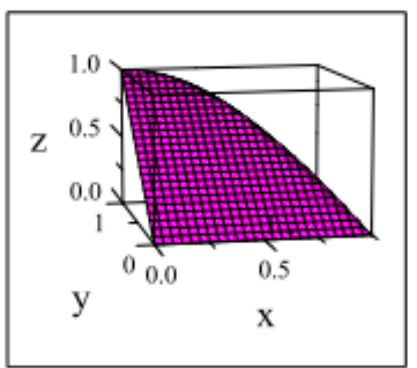

$I_{M, N_{2}}(p, q)=\min \left(q, 1-p^{2}\right)$

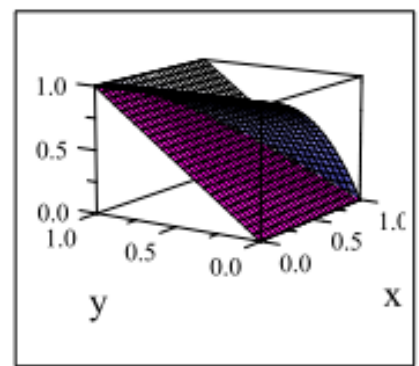

$I_{S_{M}, N_{2}}$ and $I_{M, N_{2}}$

2) A fuzzy negation $N_{3}(p)=\left\{\begin{array}{l}1 \text { if } p=0 \\ 0 \text { otherwise. }\end{array}\right.$

Then, the basic $\left(S, N_{3}\right)$ implications and $\mathrm{I}_{S_{M}, N_{3}}\left(T, N_{3}\right)$ co-implications $J_{M, N_{3}}$ are:

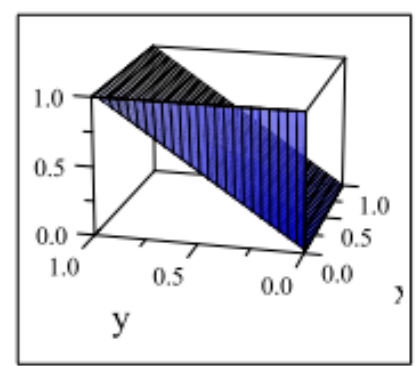

$I_{S_{M}, N_{3}}(p, q)=\left\{\begin{array}{l}1 \text { if } p \leq 0, \\ q \text { if } p>0 .\end{array}\right.$

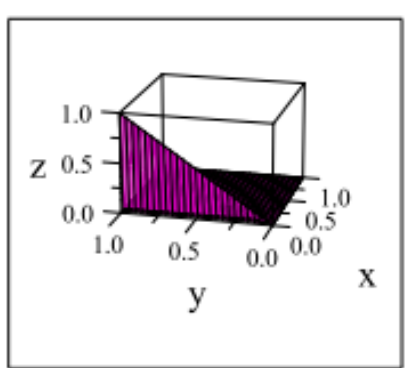

$J_{M, N_{3}}(p, q)=\left\{\begin{array}{l}q \text { if } p=0 \\ 0 \text { if } p>0\end{array}\right.$

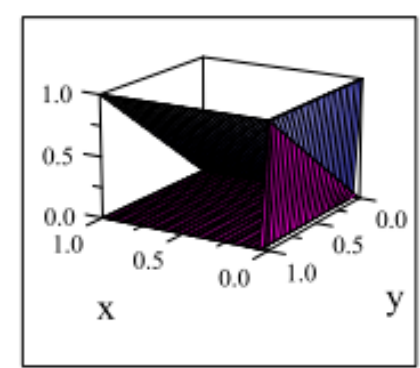

$I_{S_{M}, N_{3}}$ and $J_{M, N_{3}}$ 
As noted $\mathrm{I}_{S_{M}, N_{3}}$ is the least $\left(S, N_{3}\right)$ implications and $J_{M, N_{3}}$ is the least $\left(T, N_{3}\right)$ co-implications.

3) A fuzzy negation $N_{4}(p)=\left\{\begin{array}{l}1 \text { if } p<1, \\ 0 \text { if } p=1 .\end{array}\right.$ then, the basic $(S, N)$ implications $I_{S_{M}, N}(T, N)$ co-implications $J_{M, N}$.

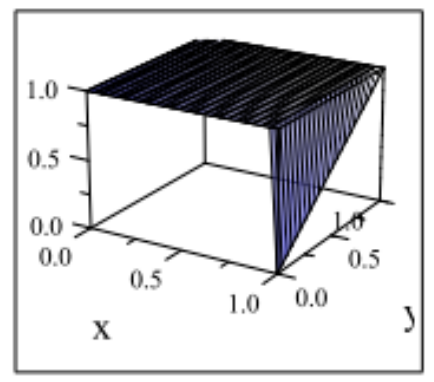

$I_{S_{M}, N_{4}}(p, q)= \begin{cases}1 & \text { if } p<1, \\ q & \text { if } p=0 .\end{cases}$

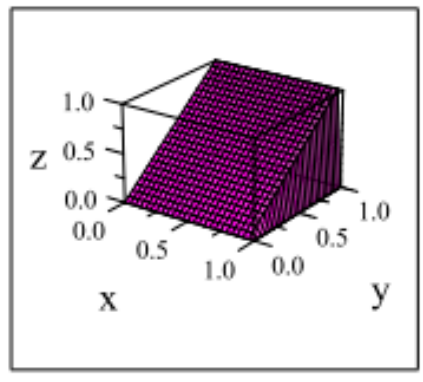

$J_{M, N_{4}}(p, q)= \begin{cases}q & \text { if } p<1 \\ 0 & \text { if } p=1\end{cases}$

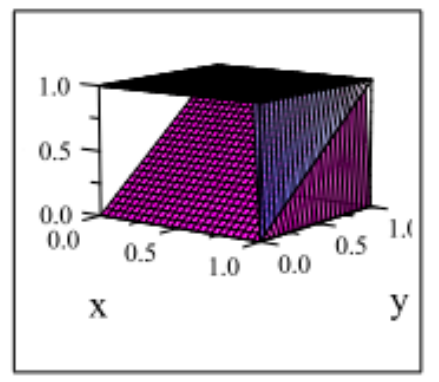

$I_{S_{M}, N_{4}}$ and $J_{M, N_{4}}$

As noted $I_{S_{M}, N_{4}}$ is the greatest $\left(S, N_{4}\right)$ implications and $J_{M, N_{4}}$ greatest $\left(T, N_{4}\right)$ co-implications.

\section{Residual Fuzzy Co-Implication in Dual Heyting}

Heyting algebra logic is the system on Heyting algebras and Brouweriaun algebras. Heyting algebra $\langle L, \wedge, \vee, \rightarrow, 0,1\rangle$ is lattice with the bottom 0 , the top 1 , and the binary operation called implication $\rightarrow$ such that, $\forall p, q, r \in L, p \rightarrow q$ is the relative pseudocomplement of a with respect to $\mathrm{c}$. That is to say

$$
p \wedge r \leq q \leftrightarrow p \rightarrow q, \quad \forall p, q, r \in L .
$$

In other words, the set of all $b \in L$ such that $p \wedge r \leq q$ contains the greatest element, denoted by $p \rightarrow q$. Precisely

$$
p \rightarrow q=\sup \{r \in L \backslash p \wedge r \leq q\} .
$$


The dual of Heyting algebra is called Brouwerian algebra $(L, \wedge, \vee, \stackrel{*}{\longrightarrow}, 0,1)$ is a lattice with 0 and 1, and the binary operation called co-implication $\stackrel{*}{\longrightarrow}$ in dual Heyting algebra. Satisfying $\forall p, q, r \in L$.

$$
p \wedge r \geq q \leftrightarrow p \stackrel{*}{\longrightarrow} q
$$

The set of all $r \in L$ such that $p \vee r \geq q$ contains the smallest element, denoted by $p \stackrel{*}{\longrightarrow} q$. Precisely

$$
p \stackrel{*}{\longrightarrow} q=\inf \{r \in L \backslash p \vee r \geq q\}
$$

Definition 4.1. Let $S$ is the t-conorm of right continuous $T$. Then, the residual co-implication ( $R^{*}$-implication) derived from $S$, is

$$
J_{S}(p, q)=\inf \{r \in[0,1] \backslash S(r, p) \geq q\}, \forall p, q \in[0,1] .
$$

$R^{*}$-implication come from residuted lattices based on residuation property $\left(R^{*} P\right)$ that can be written as

$$
S(r, p) \geq q \text { if and only if } r \geq J_{S}(p, q) .
$$

The operation $J_{S}(x, y)$ is called residual co-implication of the t-conorm $S$.

We now list the residual co-implication associated to the standard left-continuous t-norms previously introduced.

Applying the above concepts to the standard t-norms we obtain the following interesting results.

(1) Residuum of the Maximum t-conorm $S_{M}(p, q)$, is

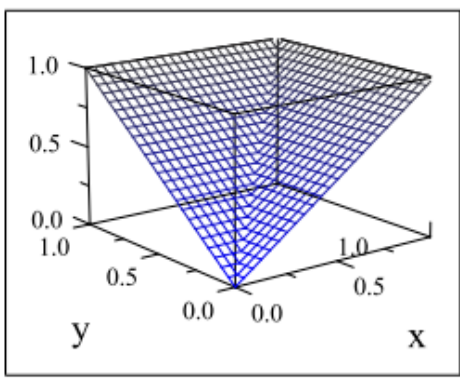

$S_{M}(p, q)$

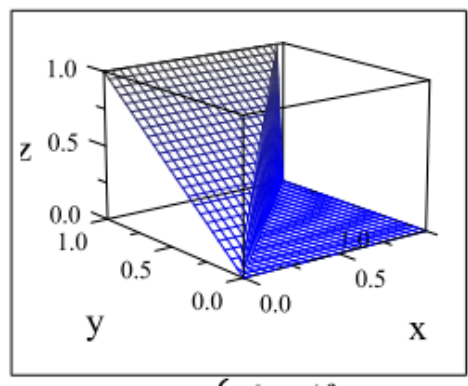

$J_{S_{M}}(p, q)= \begin{cases}0 & \text { if } p \geq q, \\ y & \text { otherwise. }\end{cases}$

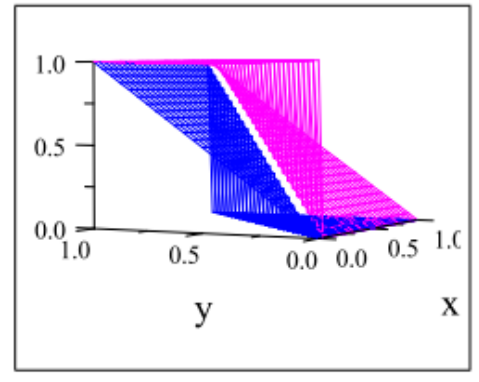

$I_{M}$ and $J_{S_{M}}$

(2) Residuum of the Probabilistic sum t-conorm $S_{\Pi}(p, q)$ is 

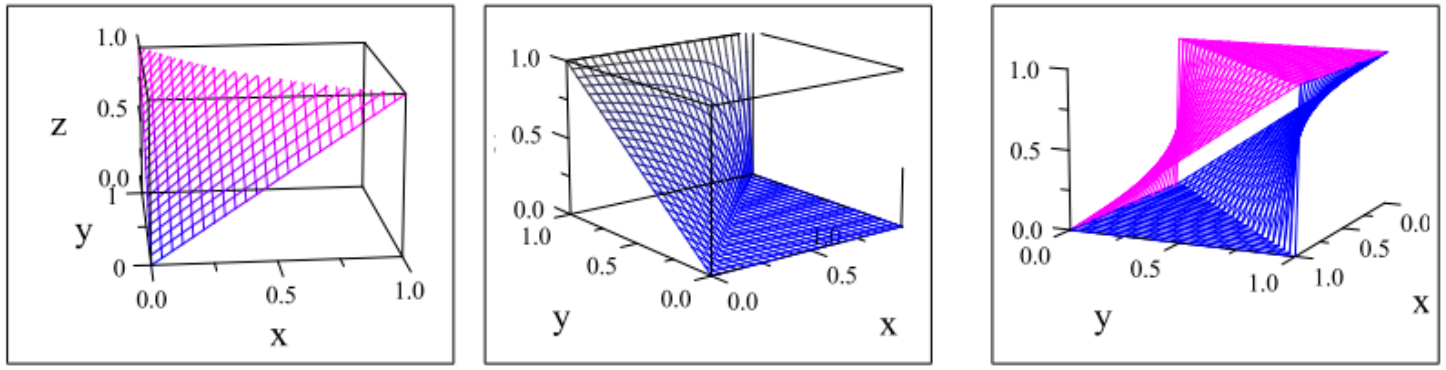

$$
S_{\Pi}(p, q) \quad J_{S_{\Pi}}(p, q)=\left\{\begin{array}{ll}
0 & \text { if } p \geq q, \\
\frac{q-p}{1-p} & \text { otherwise. }
\end{array} \quad I_{\Pi} \text { and } J_{S_{\Pi}}\right.
$$

(3) Residuum of the Bounded Sum t-conorm $S_{L}(p, q)$ is

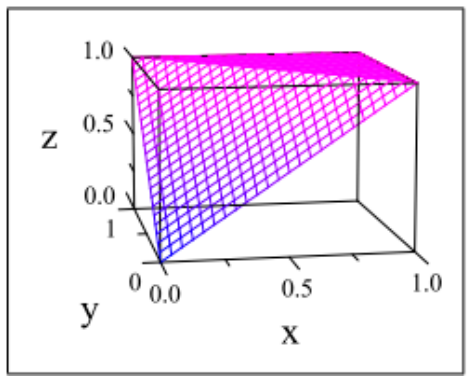

$S_{L}(p, q)$

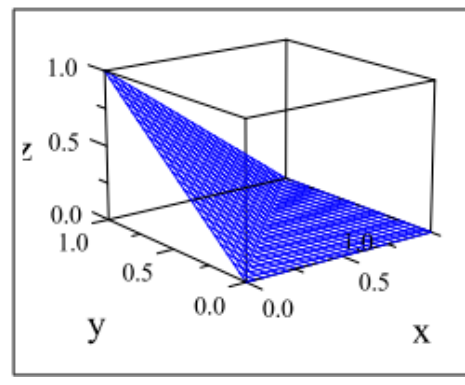

$J_{S_{L}}(p, q)=\max (0, q-p)$

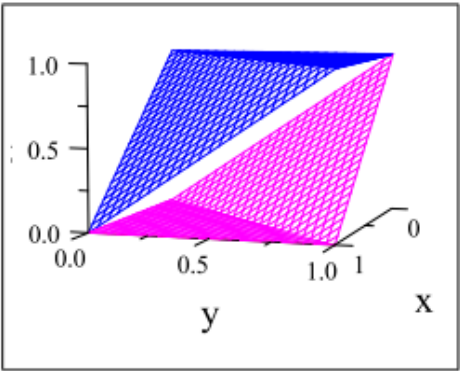

$S_{L}$ and $J_{S_{L}}$

(4) Residuum of the Nilpotent t-conorm $S_{N}(p, q)$ is
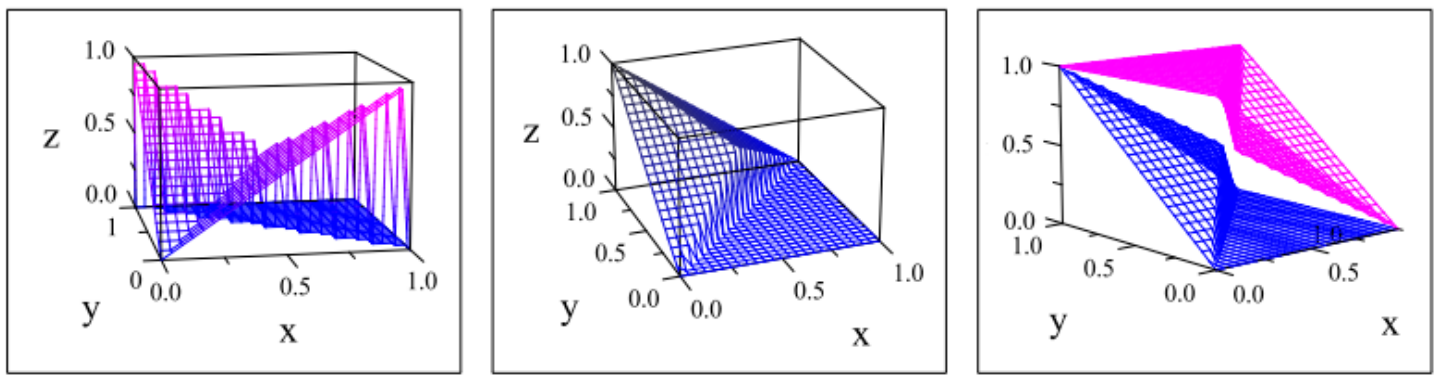

$S_{N}(p, q) \quad J_{S_{N}}(p, q)=\left\{\begin{array}{cl}0 & \text { if } p \geq q \\ \min (1-p, q) & \text { otherwise }\end{array} \quad S_{N}\right.$ and $J_{S_{N}}$

(5) Residuum of the Hamacher t-conorm $S_{H}(p, q)$ is 


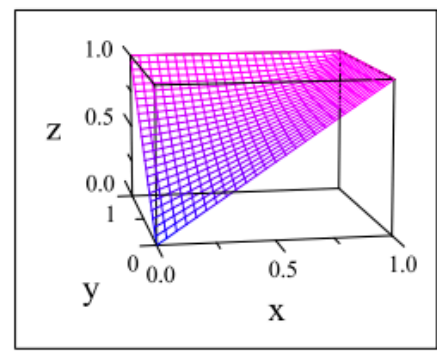

$S_{H}(x, y)$

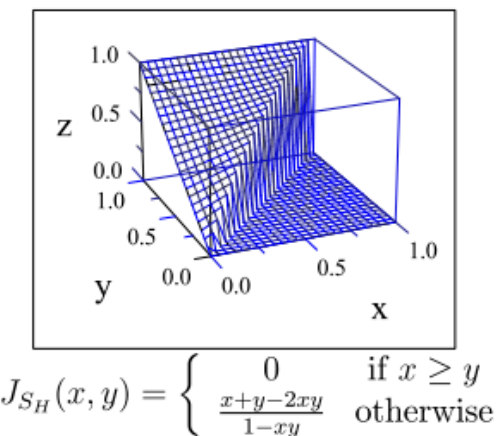

$J_{S_{H}}(x, y)=\left\{\begin{array}{cc}0 & \text { if } x \geq y \\ \frac{x+y-2 x y}{1-x y} & \text { otherwise }\end{array}\right.$

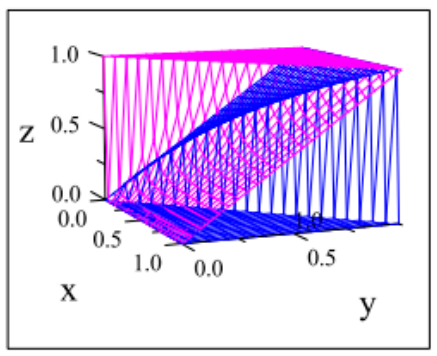

$I_{H}(x, y)$ and $J_{S_{H}}(x, y)$

(6) Residuum of the Dubois-Prade t-conorm $S_{D}(p, q)$ is

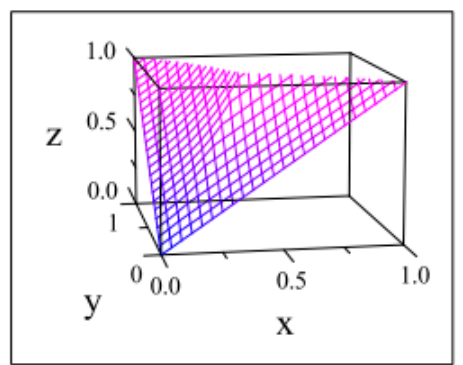

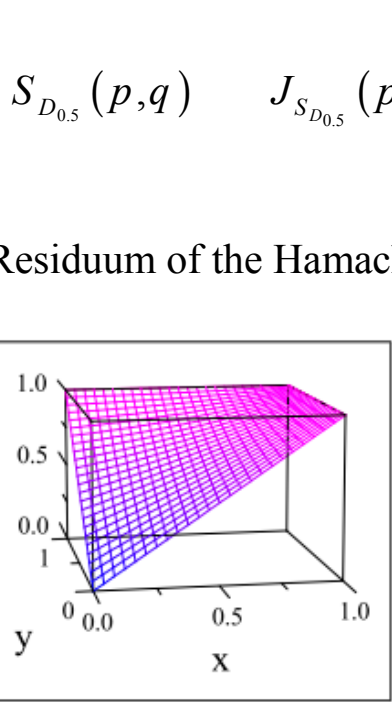

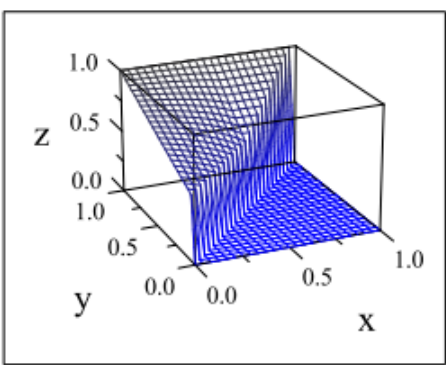

0

if $p \geq q$

if $p<q$

(7) Residuum of the Hamacher's parametric t-conorm is $S_{T_{\alpha}}(p, q)$ is

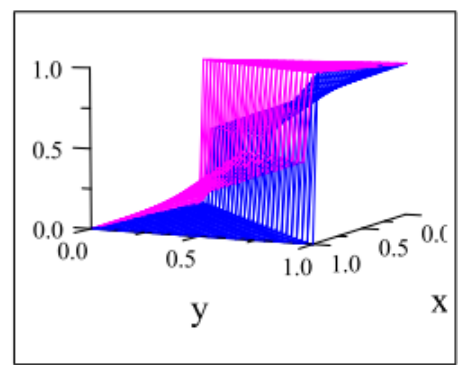

$S_{D_{0.5}}$ and $J_{S_{D_{0.5}}}$
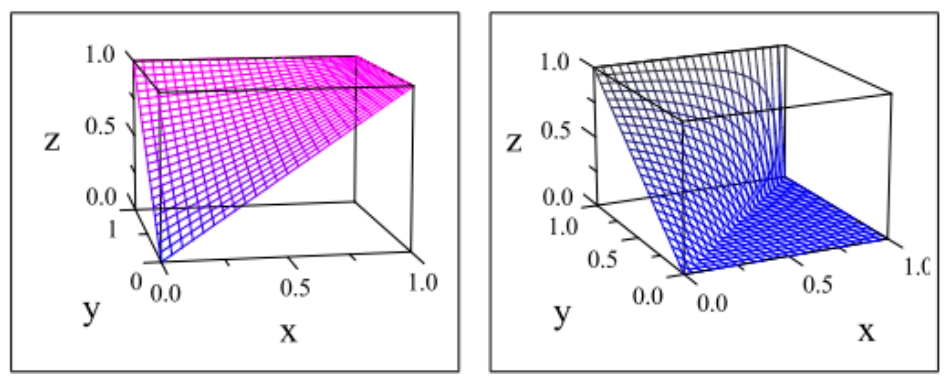

$S_{T_{\alpha}}(p, q) \quad J_{S_{T_{\alpha}}}(p, q)=\left\{\begin{array}{cc}0 & \text { if } p \geq q \\ \frac{\alpha q-p+(1-\alpha) q}{1-(2-\alpha) p+(1-\alpha) p} & \text { if } p<q\end{array} \quad S_{T_{\alpha}}\right.$ and $J_{S_{T_{\alpha}}}$

\section{$5(T, N)$ Co-Implication and Residual Co-implication Properties}

In this section we introduce some properties for $(T, N)$ co-implication and residual co-implication.

Proposition 5.1. For a left continuous t-norm $T$, then $N_{T}$ is left-continuous. 
Proof: Let $T$ be left-continuous. Assume that there exist $p, q \in[0,1]$ such that

$$
N_{T}(p)<q \leq N_{T}(p-\varepsilon), \forall \mathcal{E} \in(0, x] .
$$

This contradicts the left-continuity of $T$ and hence, $N_{T}$ must be left-continuous.

Proposition 5.2. For a left continuous t-norm $T$, then the supremum in the definition of $N_{T}$ is the maximum, i.e.,

$$
N_{T}(p)=\max \{t \in[0,1] \backslash T(p, q)=0\}, \quad \forall p \in[0,1],
$$

where the left side exists for all $p \in[0,1]$.

Proof: From the previous proposition and since $N_{T}(p) \geq N_{T}(p)$, for all $p \in[0,1]$, one has $T\left(p, N_{T}(p)\right)=0$, that means, by $N_{T}$ definition that the supremum is the maximum.

Proposition 5.3. For a left continuous t-norm $T$, then $\forall p, q \in[0,1]$ the following equivalence holds:

$$
N_{T}(p) \geq q \Leftrightarrow T(p, q)=0
$$

Proof: Suppose that $N_{T} \geq q$ for some $p \in[0,1]$ we consider two cases:

(i) $\mathrm{N}_{T}>q \Rightarrow \exists t^{\prime}>q: T\left(p, t^{\prime}\right)=0$,

$$
\Rightarrow T(p, q)=0 . \quad \text { (By monotonicity of } T \text { ) }
$$

(ii) $\mathrm{N}_{T}=q \Rightarrow q \in\{t \in[0,1] \backslash T(p, t)=0\}$,

$$
\Rightarrow T(p, q)=0 \text { or } q \notin\{\mathrm{t} \in[0,1] \backslash T(p, t)=0\} .
$$

Therefore, there exists an increasing sequence $\left(t_{i}\right)_{i \in N}$ such that $t_{i}<q$ and $T\left(p, t_{i}\right)=0$ for all $i \in N$ and $\lim _{i \rightarrow \infty} t_{i}=q$. By the left continuity of $T$ we get

$$
T(p, q)=T\left(p, \lim _{i \rightarrow \infty} t_{i}\right)=T\left(p, t_{i}\right) \lim _{i \rightarrow \infty} t_{i}=0,
$$

which is a contradiction.

On the other side assume that $T$ is a left continuous t-norm and for some $p, q \in[0,1]$.

$$
T(p, q)=0 \Rightarrow q \in\{t \in[0,1] \backslash T(p, t)=0\}
$$




$$
\begin{aligned}
& \Rightarrow q \leq \max \{t \in[0,1] \backslash T(p, t)=0\}, \\
& \Rightarrow q \leq N_{T}(p) .
\end{aligned}
$$

The proof is complete.

Proposition below states how a $(S, N)$ implications gives rise to a fuzzy $(T, N)$ co-implication and vice-versa.

Proposition 5.4. A mapping $J_{T, N}$ from $[0,1]^{2}$ into $[0,1]$ is a $(T, N)$ co- implication with strong negation iff

$$
J_{T, N}(p, q)=N\left(I_{S_{T}, N}(q, p)\right)
$$

for some $I_{S_{T}, N}$ and fuzzy (strong) negation $N$.

Conversely, $\mathrm{I}_{S_{T}, N}$ from $[0,1]^{2}$ into $[0,1]$ is a $(S, N)$ implication iff

$$
I_{S_{T}, N}(p, q)=N\left(J_{T, N}(q, p)\right)
$$

for some $J_{T, N}$ and fuzzy (strong) negation $N$.

Theorem 5.1. For t-norm $T$, then $J_{T, N} \in C o-F I$.

Proof: We have to show that $J_{1}, J_{2}$, and $J_{3}$ in definition of fuzzy co-implication are satisfied for all $p, q, r \in[0,1]$.

$$
\begin{aligned}
& J_{1}: J_{T, N}(1,1)=J_{T, N}(1,0)=J_{T, N}(0,0)=0 \text { and } J_{T, N}(0,1)=1 . \\
& J_{2}: p \leq r \Rightarrow N(p) \geq N(r) \Rightarrow T(q, N(p)) \geq T(q, N(r)) \Rightarrow J_{T, N}(p, q) \geq J_{T, N}(r, q) . \\
& J_{3}: q \leq r \Rightarrow T(q, N(p)) \leq T(q, N(p)) \Rightarrow J_{T, N}(p, q) \leq J_{T, N}(p, r) .
\end{aligned}
$$

Theorem 5.2. All $(T, N)$ co-implications are fuzzy implications satisfy (Co-NP) and (Co-EP).

Proof: If an $(T, N)$ co-implications then

$$
J_{T, N}(0, q)=T(q, 1)=q .
$$


Also,

$$
\begin{aligned}
J_{T, N}(p, J(q, r))=T\left(J_{T, N}\right. & (q, r), N(p))=T(T(r, N(q)), N(p)) \\
& =T(N(p), T(r, N(q)))=T(T(N(p), r), N(q)) \\
& =T(T(r, N(p)), N(q))=T\left(J_{T, N}(p, r), N(q)\right)=J_{T, N}(q, J(p, r)) .
\end{aligned}
$$

Theorem 5.3. If $I_{S_{T}, N}$ satisfies (IP) with strong negation then $J_{T, N}$ satisfies (Co-IP).

Proof: $J_{T, N}(p, p)=T(p, N(x))=T(N(p), p)=N(S(p, N(p)))$

$$
=N(S(N(p), p))=N\left(I_{S_{T}, N}(p, p)\right)=N(1)=0 .
$$

Theorem 5.4. If $I_{S_{T}, N}$ satisfies $(O P)$ with strong negation then $J_{T, N}$ satisfies (Co-OP).

Proof: We would like to prove that

$$
J_{T, N}(p, q)=0 \Leftrightarrow p \geq q .
$$

Let

$$
\begin{aligned}
p \geq q \Leftrightarrow N & (p) \leq N(q) \Leftrightarrow I_{S_{T}, N}(N(p), N(q))=1, \text { by }(\mathrm{OP}) \\
& \Leftrightarrow N\left(I_{S_{T}, N}(N(p), N(q))\right)=N(1) \Leftrightarrow N(S(p, N(q)))=0 \\
& \Leftrightarrow N(N(T(N(p), q)))=0 \Leftrightarrow T(q, N(p))=0 \\
& \Leftrightarrow J_{T, N}(p, q)=0 .
\end{aligned}
$$

Theorem 5.6. For t-norm $T$ and a fuzzy negation $N$, then

$$
J_{T, N}(p, p)=0, \forall p \in[0,1],
$$

iff

$$
T(p, N(p))=0, \text { for all } p \in[0,1] \text {. }
$$

Proof: If $J_{T, N}(p, p)=0, \forall p \in[0,1]$, then

$$
T(p, N(p))=J_{T, N}(p, p)=0, \forall p \in[0,1] .
$$

Conversely, if $T(p, N(p))=0, \forall p \in[0,1]$, then

$$
J_{T, N}(p, x)=T(p, N(p))=0, \forall p \in[0,1] .
$$


Theorem 5.7. For a left continuous t-norm $T$ and a continuous fuzzy negation $N$, then

$$
J_{T, N}(p, p)=0, \forall p \in[0,1],
$$

iff

$$
N(p) \leq N_{T}(p), \forall p \in[0,1] .
$$

Proof: Let $T$ is a left continuous, t-norm and for a continuous fuzzy negation $N$, then

$$
N_{T}(x)=\max \{t \in[0,1]: T(p, t)=0\}, \forall p \in[0,1] .
$$

Then $J_{T, N}(p, p)=T\left(p, N_{T}(p)\right)=0$.

By monotonicity of $T$ and if

$$
N(p) \leq N_{T}(p)
$$

then $T(p, N(p))=0$.

Conversely, let $J_{T, N}(p, p)=0, \forall p \in[0,1]$, then $T(p, N(p))=0, \forall p \in[0,1]$ and if

$$
N(p) \in\{t \in[0,1]: T(p, t)=0\}
$$

then

$$
N(p) \leq \max \{t \in[0,1]: T(p, t)=0\}=N_{T}(p) .
$$

Theorem 5.8. For a right continuous t-conorm $S$, then $J_{S} \in C o-F I$.

Proof: We have to show that $J_{1}, J_{2}$, and $J_{3}$ in definition of fuzzy co-implication are satisfied for all $p, q, r \in[0,1]$.

$$
\begin{gathered}
J_{1}: J_{S}(1,1)=\inf \{r \in[0,1] \backslash S(r, 1) \geq 1\}=0, \\
J_{S}(1,0)=\inf \{r \in[0,1] \backslash S(r, 1) \geq 0\}=0, \\
J_{S}(0,0)=\inf \{r \in[0,1] \backslash S(r, 0) \geq 0\}=0, \\
J_{S}(0,1)=\inf \{r \in[0,1] \backslash S(r, 0) \geq 1\}=1 . \\
J_{2}: p \leq r \Rightarrow\{t \in[0,1] \backslash S(t, p) \geq q\} \subseteq\{t \in[0,1] \backslash S(t, r) \geq q\} \\
\Rightarrow \inf \{t \in[0,1] \backslash S(t, p) \geq q\} \geq \inf \{t \in[0,1] \backslash S(t, r) \geq q\} \\
\Rightarrow J_{S}(p, q) \geq J_{S}(r, q) .
\end{gathered}
$$




$$
\begin{aligned}
J_{3}: q \leq r & \Rightarrow\{t \in[0,1] \backslash S(t, p) \geq q\} \supseteq\{t \in[0,1] \backslash S(t, p) \geq r\} \\
& \Rightarrow \inf \{t \in[0,1] \backslash S(t, p) \geq q\} \leq \inf \{t \in[0,1] \backslash S(t, p) \geq r\} \\
& \Rightarrow J_{S}(p, q) \leq J_{S}(p, r) .
\end{aligned}
$$

Theorem 5.9. A co-implications $J_{S}$ satisfy (Co-NP) and (Co-IP).

Proof: For any $S$ t-conorm and $p, q \in[0,1]$ we get

$$
J_{S}(0, q)=\inf \{r \in[0,1] \backslash S(r, 0) \geq q\}=\inf \{r \in[0,1] \backslash r \geq q\}=q
$$

Also,

$$
J_{S}(p, p)=\inf \{r \in[0,1] \backslash S(r, p) \geq p\}=0 .
$$

Theorem 5.10. If $S$ is a right continuous, then $J_{S}$ satisfy (Co-EP) and Co-OP).

Proof: For any right continuous t-conorm $S$ and for all $p, q, r \in[0,1]$ and by using $R^{*}$ condition we have

$$
\begin{aligned}
J_{S}\left(p, J_{S}(q, r)\right)=\inf \{t & {[0,1] \backslash S(t, p) \geq\} J_{S}(q, r)=\inf \{t \in[0,1] \backslash S(S(t, p), q) \geq r\} } \\
& =\inf \{t \in[0,1] \backslash S(t, S(p, q)) \geq r\}=\inf \{t \in[0,1] \backslash S(t, S(q, p)) \geq r\} \\
& =\inf \{t \in[0,1] \backslash S(S(t, q), p) \geq r\}=\inf \left\{t \in[0,1] \backslash S(t, q) \geq J_{S}(p, r)\right\} \\
& =J_{S}\left(q, J_{S}(p, r)\right) .
\end{aligned}
$$

Now, we would like to prove that

$$
J_{S}(p, q)=0 \Leftrightarrow p \geq q
$$

If $p \geq q$ then $S(p, 0)=p \geq q$, so $J_{S}(p, q)=0$. Conversely, if $J_{S}(p, q)=0$, then because of $R^{*}$ condition we get $S(p, 0) \geq q$, i.e., $p \geq q$.

\section{Conclusion}

There is four usual models of fuzzy implications that is $(S, N)$, residual, QL-operation $(I(p, q)=S(N(p), T(p, q)), p, q \in[0,1]$, and D-operations $(I(p, q)=S(T(N(p), N(q)), q)$ $, p, q \in[0,1]$ implication. In this paper we introduced $(T, N)$ and residual co-implication. Now, an interesting natural questions arises that to find Co-QL-operation and Co-D-operations.

\section{Competing interests}

The authors declare that they have no competing interests. 


\section{References}

[1] E.E. Kerre, C. Huang, and D. Ruan. Fuzzy Set and Approximate Reasoning. Wu Han University Press, Wu chang, 2004.

[2] M. Mas, M. Monserrat, J. Torrens, and E. Trillas. A survey on fuzzy implication functions. IEEE Transactions on Fuzzy Systems, 15(6):1107, 1121, 2007.

[3] S. Gottwald. ATreatise on Many-Valued Logic. Resarch Studies Press, Baldok, 2001.

[4] L. Tsoukalas, R. Uhring, and L. Zadeh. Fuzzy and Neural Approaches in Engineering. Adaptive and Learning Systems for Signal Processing, communications and control. Wiley-Interscience, New York, 1997. [5] E.P. Klement, R. Mesiar and E. Pap, Triangular Norms, Kluwer, Academic Publisher, Dordrecht, 2000.

[6] Weber, S., A General Concept of Fuzzy Connectives, Negations and Implications Based on T-norms and T-conorms, Fuzzy Sets and Systems 11, pp. 115-134, 1983.

[7] B. De Baets, Coimplicators, the forgotten connectives, Tatra Mountains Mathematical Publications, 12: 229-240, Tatra Mt. Math. Publ., 1997.

[8] Oh, K., Kandel, A.: Coimplication and its applications to fuzzy expert systems. Information Sciences 56, 247-260, 1991.

[9] F. Wolter, On Logics with Coimplication, Journal of Philosophical Logic, 27 (4), 353 -387, 1998.

[10] B. Schweizer and A. Sklar, Probabilistic Metric Spaces, North Holland, Amsterdam, 1983, 2nd edition: Dover Publications, Mineola, NY, 2006.

[11] I. Jebril, Mohd. S. Md. Noorani, and A. Saari, An example of a probabilistic metric space not induced from a random normed space. Bull. Malays Math. Sci. Soc. 2 (26), 93-99, 2003.

[12] M.M. Gupta and J. Qi, Theory of T-norms and fuzzy inference methods, Fuzzy Sets and Systems, 40, 431-450, 1991.

[13] E.P. Klement and R. Mesiar, Triangular Norms, Tatra Mountains Math. Publ. 13 (1997), 169-174.

[14] Fodor, J., Left-continuous t-norms in fuzzy logic: an overview. Journal of applied sciences at Budapest Tech Hungary 1(2), 2004.

[15] J.C. Fodor, M. Roubens, Fuzzy Preference Modelling and Multicriteria Decision Support, Kluwer, Dordrecht, 1994.

[16] K.C. Maes and B. De Baets. A contour view on uninorm properties.Kybernetika 42, 303-318, 2006.

[17] M. Baczynski, B. Jayaram, Fuzzy Implications, Studies in Fuzziness and Soft Computing, Vol. 231, Springer, Heidelberg, 2008.

[18] M. Baczynski, B. Jayaram, (S,N)- and R-implications: a state-of-the-art survey, Fuzzy Sets Syst. 159, 8361859, 2008.

[19] P. Li and S. Fang, A survey on fuzzy relational equations, part I: classification and solvability, Fuzzy Optimization and Decision Making, 8, 179-229, 2009.

[20] Youg Su and Zhuden Wang, Constructing implications and coimplication on a complete lattice, Fuzzy Sets Syst. 247, 68-80, 2014.

(C) 2016 by the authors; licensee Preprints, Basel, Switzerland. This article is an open access article distributed under the terms and conditions of the Creative Commons by Attribution (CC-BY) license (http://creativecommons.org/licenses/by/4.0/). 